\title{
Age and size-selective predation on larval fishes: the bigger-is-better hypothesis revisited*
}

\author{
Matthew K. Litvak**, William C. Leggett \\ Department of Biology, McGill University, 1205 Ave. Dr. Penfield, Montreal, Quebec, H3A 1B1, Canada
}

\begin{abstract}
A combined mesocosm and microcosm approach was employed to analyze the impact of age, and of size independent of age effects, of yolk-sac capelin larvae Mallotus villosus on their vulnerability to predation. The impact by both visual (3-spined stickleback Gasterosteus aculeatus) and non-visual (jellyfish Staurophora mertensi) predators was evaluated. We found, as predicted from the literature, that the probability of capture by both predator types decreased with larval age. However, contrary to the current view, when the predators were offered a mixture, and hence a choice, of larvae separated in age by $2 \mathrm{~d}$, the probability of death by predation was higher for older larvae. We also examined size effects, independent of age effects, by generating large and small larvae of identical age through artificial fertilization of eggs from selected capelin females. We found no difference in the probability of capture of small versus large larvae of the same age. Yet, when the predators were presented with a mixture, and hence a choice of small versus large larvae, the larger larvae experienced higher mortality. This, too, is counter to current thinking. We show that being smaller at a given age may actually confer a survival advantage to larvae. These outcomes are discussed in terms of the interacting probabilities of encounter, attack and capture which define the probability of larval death due to predation.
\end{abstract}

\section{INTRODUCTION}

Knowledge of the size- and age-specific vulnerability of larval fishes to predation and starvation is central to any attempt to understand the basis of year class formation and recruitment (Folkvord \& Hunter 1986). Since starvation contributes directly to mortality only after the transition to exogenous feeding, predation must dominate as a source of mortality while yolk-sac energy reserves are intact. In many marine fish species, particularly those of commercial importance, most individuals die before feeding is established (Hewitt et al. 1985, Ware \& Lambert 1985, McGurk 1986, Taggart \& Leggett 1987). Reports of mortality rates of yolk-sac larvae can be extremely high. For example, yolk-sac larvae of capelin Mallotus villosus and jack mackerel Trachurus symmetricus are reported to experience mortality rates as high as $60 \%$

\footnotetext{
- Contribution to the programs of the Huntsman Marine Science Centre and the Groupe Interuniversitaire de Recherches Oceanographiques du Quebec

- Present address: Huntsman Marine Science Center, Brandy Cove Road, St. Andrews, N.B. EOG 2X0, Canada
}

$\mathrm{d}^{-1}$ (Taggart \& Leggett 1987) and $80 \% \mathrm{~d}^{-1}$ (Hewitt et al. 1985) respectively.

Fish larvae face predation from a wide variety of predators which employ different capture strategies. Ichthyoplankton predators are known to include both invertebrates and vertebrates (for reviews see Hunter 1984, Bailey \& Houde 1989). There is, however, a general lack of information on the impact of such predators on populations of fish larvae (Fancett \& Jenkins 1988) and, in particular, on the relationship between larval size, age, or development and the vulnerability to predation (Bailey \& Houde 1989).

It has long been surmised that the risk of death due to predation changes dramatically during the first few days after hatching. This is believed to be due to the rapid increase in length and/or changes in behaviour related to development during this period (Bailey \& Batty 1984, Blaxter \& Batty 1985, Blaxter 1986, Eaton \& DiDomenico 1986, Webb \& Weihs 1986, Butler \& Pickett 1988, Fuiman 1989). The view most commonly expressed in the literature is that larger and older larvae are less vulnerable to predation (Bailey \& Batty 1984, Blaxter \& Batty 1985, Blaxter 1986, Eaton \& DiDomenico 1986, Webb \& Weihs 1986, Butler \& Pick- 
ett 1988, Miller et al. 1988, and others). This concept, particularly regarding the importance of being larger at any given age, is now approaching the status of a paradigm.

Most experimental studies of predation on larval fishes conducted to date have focused solely on the probability of capture of larvae of a single size or age, thereby ignoring the potential importance of encounter rate and the choice of which larva to attack (large versus small, old versus young). However, it is the product of the individual probabilities of location, pursuit, attack, and capture of prey which determine the outcome of a predation event (O'Brien 1979).

The restriction of previous analyses of potential for predation on larvae to a single size or age cohort is also an unrealistic portrayal of nature. In nature, predators are rarely, if ever, confronted with larvae of a single size or age. As larvae hatch they can form heterogeneous mixtures of individuals of different ages. In addition to this variation in the age of a recently emerged group of larvae, there exists variation in size within each age cohort. For example, previous research on capelin Mallotus villosus has revealed great variability in the sizes of larvae produced by different females (Chambers et al. 1989). Thus, predators of Iarval capelin will encounter larvae of a wide range of ages and sizes. Moreover these differences will persist, and perhaps become even greater, throughout the larval period.

The experimental designs used to explore the vulnerability of larval fishes to predation have commonly compounded the effects of age and size. Larvae of different ages have routinely been used to obtain the range of larval size required to assess the relationship between size and vulnerability (Folkvord \& Hunter 1986, Butler \& Pickett 1988, Margulies 1989). In reality what has been examined in such studies is the combined influence of age (development) and size on the probability of capture. Given that there can be considerable variation in size within a like-aged cohort (Chambers et al. 1989), it is important, when examining the impact of prey selection on the probability that an individual larva will survive to the next stage, to view the effect of age as a reflection of the significance of timing on survival.

For example, the temporal and spatial overlap in predator and prey fields can be phase-shifted if the timing of hatching, emergence, or the onset of pelagic drift occurs during times of low predator densities (Frank \& Leggett 1981a, b, 1982, Leggett et al. 1984). When predator/prey overlap does occur, either older or younger larvae may be preferred as prey. Under such scenarios it is likely that individuals which were born at the 'right' window in time will survive. Moreover, if size-selective predation occurs within such an age cohort, individuals of the 'right' size will have a higher probability of survival.

The current view that, within a cohort or year class of larval fish, larger larvae will have a higher probability of survival (the 'bigger-is-better' hypothesis) is based almost exclusively on experiments that are devoid of predator choice or that have assumed that size and age are equivalent.

The goal of the experiments reported here was to critically examine the relationship between vulnerability to predation and larval age and size in greater detail. We present the results of a series of experiments on the vulnerability of yolk-sac larval capelin Mallotus villosus of different ages, and of different sizes within ages, to predation. We employed a combined mesocosm (enclosure) and microcosm (lab tanks) approach Both visual (vertebrate) and non-visual (invertebrate) predators were used. We conclude that, when predators are provided with a choice of yolk-sac larvae of different ages or sizes (independent of age effects), both older and larger larvae are more vulnerable to predation. This is contrary to the current hypothesis. We also discuss circumstances in which being smaller at age may act as a refuge against predation. More simply put, at times, the probability that a larva will survive predation may be enhanced if it is younger or smaller

\section{METHODS AND MATERIALS}

The study was conducted at Bryant's Cove $\left(47^{\circ} 40.5^{\prime}\right.$ N, $\left.53^{\circ} 11.0^{\prime} \mathrm{W}\right)$, Conception Bay, Newfoundland, an important spawning site for capelin (for further details on the site see Frank \& Leggett 1981a, b, 1982). The in situ enclosure experiments were performed during summer 1989, the laboratory experiments during summer 1990.

Larvae and predators. Capelin larvae were acquired from 2 sources. Some larvae were collected from Bryant's Cove beach at the time of hatching and held in the laboratory at $8^{\circ} \mathrm{C}$ for later use. Larvae were also produced by artificially fertilizing eggs obtained from wild adults and incubating them at $8^{\circ} \mathrm{C}$. Individual larvae were collected at hatching and maintained at $8{ }^{\circ} \mathrm{C}$ until required for the experiments.

The non-visual predators, jellyfish Staurophora mertensi, were collected by SCUBA from Bryant's Cove immediately prior to the experimental trials. The visual predators, sea-run 3-spine stickleback Gasterosteus aculeatus, were captured with seine nets at the mouth of South River (10 km south of Bryant's Cove) several days before the experiments were initiated. The sticklebacks were kept in a flow-through tank system supplied with seawater pumped directly from Bryant's 
Cove. All sticklebacks were held for at least $3 \mathrm{~d}$ prior to their use in experiments.

Enclosure experiments. These trials were conducted in sixteen, $3.2 \mathrm{~m}^{3}$ black Dacron-walled in situ enclosures [see de Lafontaine \& Leggett $(1987 \mathrm{a}$, b) for details of enclosure design and an evaluation of their effectiveness for such studies]. These experiments assessed the influence of larval age on vulnerability to predation by both visual and non-visual predators.

Each of the 16 enclosures was stocked with 640 capelin larvae (200 larvae $\mathrm{m}^{-3}$ ) collected from the beach at Bryant's Cove. To produce larvae of different ages we collected a group of newly hatched larvae $2 \mathrm{~d}$ prior to the initiation of experiments. These larvae were held in ambient-temperature seawater pumped from Bryant's Cove. On the day of the experiment we collected a second group of newly hatched larvae. We counted 320 newly hatched and 320 two-day-old larvae individually into mason jars containing Cove seawater at ambient temperatures. These jars were then suspended in the enclosures for at least $1 \mathrm{~h}$ before the larvae were gently released by inverting the submersed jar. The larvae were allowed to acclimate and disperse in the enclosures for $4 \mathrm{~h}$ before the predation trials were initiated.

At time 0 a single predator was added to each of the enclosures that had been randomly designated for the predation treatments. Jellyfish were held in jars floated in the enclosures for 1 to $2 \mathrm{~h}$ prior to their release. Sticklebacks were placed into large plastic bags containing water and air and floated in the enclosures for $2.5 \mathrm{~h}$ before release.

In enclosure experiments involving sticklebacks, predators were stocked into 8 of the 16 enclosures. The remaining 8 served as controls. In experiments involving jellyfish we stocked predators into 12 of 16 en- closures, the remaining 4 serving as controls. Enclosure trials involving both jellyfish and sticklebacks lasted for $40 \mathrm{~h}$. We performed 2 series of experiments using stickleback predators and 1 with the jellyfish predators

At the termination of the experiments the predators were captured with an aquarium net attached to a long pole and were immediately preserved in $10 \%$ buffered formalin. The enclosures were then slowly lifted by hand and the remaining larvae were washed into the cod-end as lifting progressed. The cod-end contents were washed into a mason jar and were immediately preserved with $10 \%$ buffered formalin.

Laboratory experiments. We conducted 4 experiments in the laboratory (Table 1). Two experiments (Expts 1 and 2) provided the predators with a choice of prey size and age, the remaining two (Expts 3 and 4) involved no choice. These experiments examined the influence of: predator choice on the vulnerability of larvae of 2 different ages to predation (Expt 1); predator choice on the vulnerability of larvae of like age but of 2 different sizes to predation (Expt 2); larval age on the probability of capture (Expt 3); and larval size (independent of age effects) on the probability of capture (Expt 4). Low in situ abundances of jellyfish in Bryants Cove during summer 1990 forced us to use only the stickleback as predators in the laboratory experiments.

Lab experiments on predator/prey interactions have been criticized for biases resulting from the influence of experimental volume on mortality rates (de Lafontaine \& Leggett 1987b). To evaluate the importance of this potentially confounding effect we repeated the in situ age/choice trials in the laboratory (Expt 1 above). We assumed that if the results of the age/choice trials in the laboratory were similar to those from the mesocosm trials we could interpret the laboratory results with confidence.

Table 1. Description of experiments on predation by Gasterosteus aculeatus on larval Mallotus villosus, conducted in laboratory microcosms

Experiment Experimental treatment

$\begin{array}{ll}\text { Design } & \text { Larval age } \\ & \text { replicates }\end{array}$

$\begin{array}{cc}\text { No. of replicates } & \text { Total no. } \\ \text { for each } & \text { of trials } \\ \text { age group } & \end{array}$

age group
(1) Influence of predator choice on vulnerability of larvae of different ages to predation

(2) Influence of predator choice on vulnerability of larvae of the same age but different size to predation

(3) Influence of larval age on probability of capture

(4) Influence of larval size, independent of age, on probability of capture
Choice: 75 old with 75 young larvae (separated in age by $2 \mathrm{~d}$ )

Choice: 75 small with 75 large larvae of the same age

No choice: 150 larvae of the same age cohort

No choice: 150 large larvae of the same age or 150 small larvae of the same age
$0 \& 2,1 \& 3,2 \& 4$

8

40

$3 \& 5,4 \& 6$

$0,1,2,3,4,5$

$6-8$

38

$0,1,2,3,4,5$,

$7-10$

75

$6,7,8$

$0,2,4,6,9$

$5-6$

57 


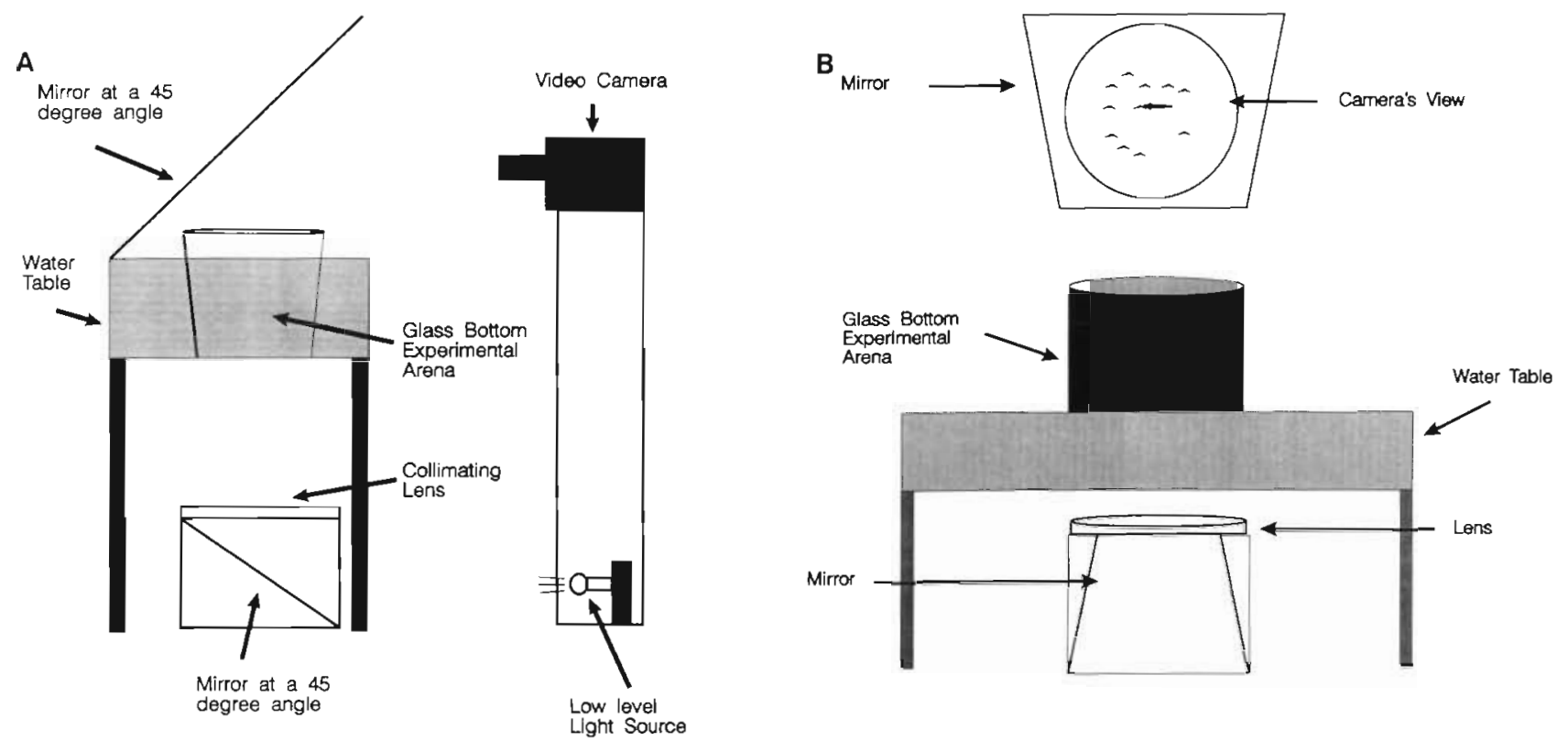

Fig. 1. (A) Side view and (B) front view of collimated light source and video system employed in the experiments described. Light travels from an underrun dissecting light bulb controlled by a veriac rheostat. The light is collimated by the lens and passes through the experimental arena to the camera. Objects in the arena which interfere with light passage will appear as a silhouette

Rearing procedures. Examination of age-specific mortality (Expts 1 and 3) involved the use of different age 'cohorts' of capelin hatchlings in each experiment. We used wild larvae collected from the beach for these experiments. The protocol for age separation matched that used in the enclosure trials.

Examination of size-specific mortality (Expts 2 and 4) involved the use of 2 'cohorts' of capelin hatchlings in each experiment. Chambers et al. (1989) showed that size at hatching is positively correlated with yolk-volume among females, and that over $71 \%$ of the overall variance in yolk-volume was due to among-female differences. We exploited this variability to create different larval size cohorts of like age.

Mature males and gravid female capelin were collected with beach seines in Bryant's Cove. A small aliquot of eggs was taken from each of 20 females and was fertilized. Yolk diameter was determined for 40 fertilized eggs from each family. We produced larvae of significantly different sizes by selecting eggs from females whose egg size distribution lay at the extremes of the total distribution for the 20 females initially sampled. The eggs were incubated at $8^{\circ} \mathrm{C}$. We conducted 4 sets of crosses over summer 1990 to generate sufficient larvae for the experiments.

Experimental protocol. The laboratory experiments were conducted in cylindrical PVC experimental arenas (Fig. 1A, B) placed in water tables equipped with a flow through system supplied with water pumped directly from Bryant's Cove. For the experiments involving single size and age cohorts (Expts 3 and 4) we used a silhouette videographic system to enhance the precision of observations of predator and prey behaviour. We did not use silhouette videography for the predator choice experiments (1 and 2) because in these experiments we were interested in the size distribution of the surviving larvae after they had been exposed to a predator and not in the details of the predatory encounters that produced that distribution.

In all 4 of the laboratory experiments (both choice and no choice experiments) larvae were placed into a single cylindrical experimental arena like that shown in Fig. 1A, B. The larvae were allowed $10 \mathrm{~min}$ to disperse. A single stickleback predator was then added to the arena. The total number of bites by the stickleback was recorded with an event recorder program written in Basic for a lap-top PC. The experiment was terminated after the stickleback had taken 75 bites or had fed for $10 \mathrm{~min}$, whichever came first. For laboratory Expts 1 and 2, at least 2 control trials were run without predators for comparison of distributions with the treatment (predator) trials. The experimental protocols for each of the laboratory experiments are summarized in Table 1.

Data acquisition and analysis. We compared the length distributions of the capelin larvae surviving the control and predator treatments in the choice experiments (enclosure trials and laboratory Expts 1 and 2) to assess the role of age and size at age on the vulnerability of capelin larvae to predation

The number of larvae recovered from each experiment was determined. We measured at least 100 larvae 


\section{Curl Factor}

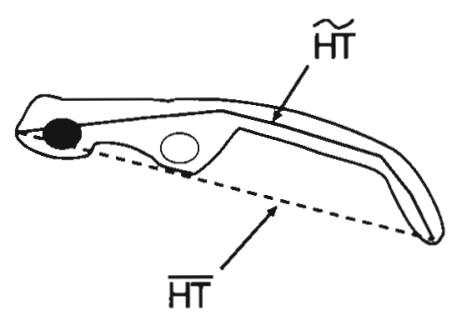

Curr Factor $=\operatorname{Arc} \operatorname{cosin} \theta(\overline{H T} / \widetilde{H})$

Fig. 2. The curl factor, an index of the degree to which individual larvae are curled due to death and of the probability that death occurred prior to the termination of the experiment and preservation, where $\widetilde{\mathrm{HT}}=$ standard length of the larva and $\overline{\mathrm{HT}}=$ euclidian distance from the coordinate origins of the standard length measurement

recovered from each in situ enclosure. All larvae surviving the laboratory choice experiments were measured. Larvae were measured with a video frame grabber system linked to a dissecting microscope. Two sets of measurements were made on each larva: (1) the standard length of the larvae measured as a polyline. and (2) the coordinates of the anterior-most point of the head and the posterior-most point of the notochord The distance between the coordinates of the head and notochord were calculated with a program written in APL (STSC* APLPLUS).

These 2 separate measures were used to classify those larvae which were alive and those which were dead at the termination of each experiment. This information is vital for the correct interpretation of the results of field and laboratory experiments of the type described (and also of field collections) because larvae shrink both as a result of the preservation process and due to the death. Radtke (1990) reported that larvae of cod Gadus morhua lose up to $15 \%$ of their body length immediately following death. Hence, if larvae which died prior to the termination of the experiments are included in the analysis, the number of small larvae in the recovered sample is positively biased.

In independent experiments we found that capelin larvae can lose from 10 to $20 \%$ of their length due to shrinkage at death and a significant loss in size due to shrinkage can occur within 5 min after death. However, when larvae were killed quickly by formalin preservation they did not exhibit such shrinkage.

We therefore evaluated the form and magnitude of shrinkage due to death with the objective of using differences as a means of distinguishing between larvae that were alive or dead at the termination of the experiment. We found that larvae which die prior to preservation exhibit significantly greater curvature then do larvae that are preserved alive and that this difference can be reliably used to distinguish between the two. We have called this distinguishing feature the curl factor, CF (Fig. 2) There was no significant difference in the CF for larvae of different ages used in this study when preserved alive. We used the upper confidence limit (95\%) for the CF of the larvae preserved alive in this experiment as the classification criterion $\left(0.297\right.$ radians or $\left.18^{\circ}\right)$ for larvae that were alive at the time of preservation and hence at the end of our enclosure and microcosm experiments. Only larvae that were classified as having been alive at the termination of the experiments were used in the analyses.

In Expts 3 and 4, which evaluated the influence of age and size on the probability of capture, the number of larvae remaining after the termination of the experiment was counted. Since the number of bites was known, it was possible to calculate the number of larvae eaten per bite (probability of capture after an attack).

We calculated the daily instantaneous mortality rates $(Z)$ inflicted on the larvae by stickleback and jellyfish predators in the enclosures using the following equation:

$$
Z=\left[\left(\ln N_{1} / N_{\mathrm{p}}\right)-\left(\ln N_{1} / N_{\mathrm{c}}\right)\right] / T
$$

where $N_{\mathrm{l}}=$ the initial number of larvae stocked, $N_{c}$ and $N_{\mathrm{p}}=$ the number of larvae recovered from the controls and the predator treatments, respectively, and $T=$ the time of experiment expressed in days (Royama 1971 , Rogers 1972, de Lafontaine \& Leggett 1988). Following de Lafontaine \& Leggett (1988) we calculated the daily predation rates $(I)$ as:

$$
I=N_{\mathrm{i}} \times[1-\exp (-\mathrm{Z})]
$$

\section{RESULTS}

\section{Enclosures}

The mean size of the sticklebacks used in the enclosures was $3.8 \pm 0.5(\mathrm{SE}) \mathrm{cm}$ and that of the jellyfish used in the enclosures was $7.7 \pm 0.3 \mathrm{~cm}$. A high percentage of the larvae stocked into the control enclosures were recovered [stickleback experiments mean $92 \pm 2 \%$ (SE); jellyfish experiments mean $98 \pm 1 \%$ l.

\section{Stickleback predators}

All data from the enclosure experiments were normal and homoscedastic. We tested for a difference between controls and treatments across the 2 experimental runs for the following variables: (1) the number of larvae recovered; (2) the proportion classified dead at the termination of the experimental trials (after arcsin 
Table 2. Gasterosteus aculeatus preying on Mallotus villosus. Enclosure age/choice trials with sticklebacks as predators. Two-way ANOVA

\begin{tabular}{|lllll}
\hline Dependent variable & Source & df & Tail & Probability \\
\hline Number recovered & Experiment & 1 & 1 & 0.116 \\
& Treatment & 1 & 1 & 0.0001 \\
Mean size & Interaction & 1 & 1 & 0.010 \\
& Experiment & 1 & 1 & 0.301 \\
& Treatment & 1 & 1 & 0.015 \\
Percent classified dead (arcsin) & Interaction & 1 & 1 & 0.087 \\
& Experiment & 1 & 1 & 0.172 \\
& Treatment & 1 & 1 & 0.397 \\
& Interaction & 1 & 1 & 0.388 \\
\hline
\end{tabular}

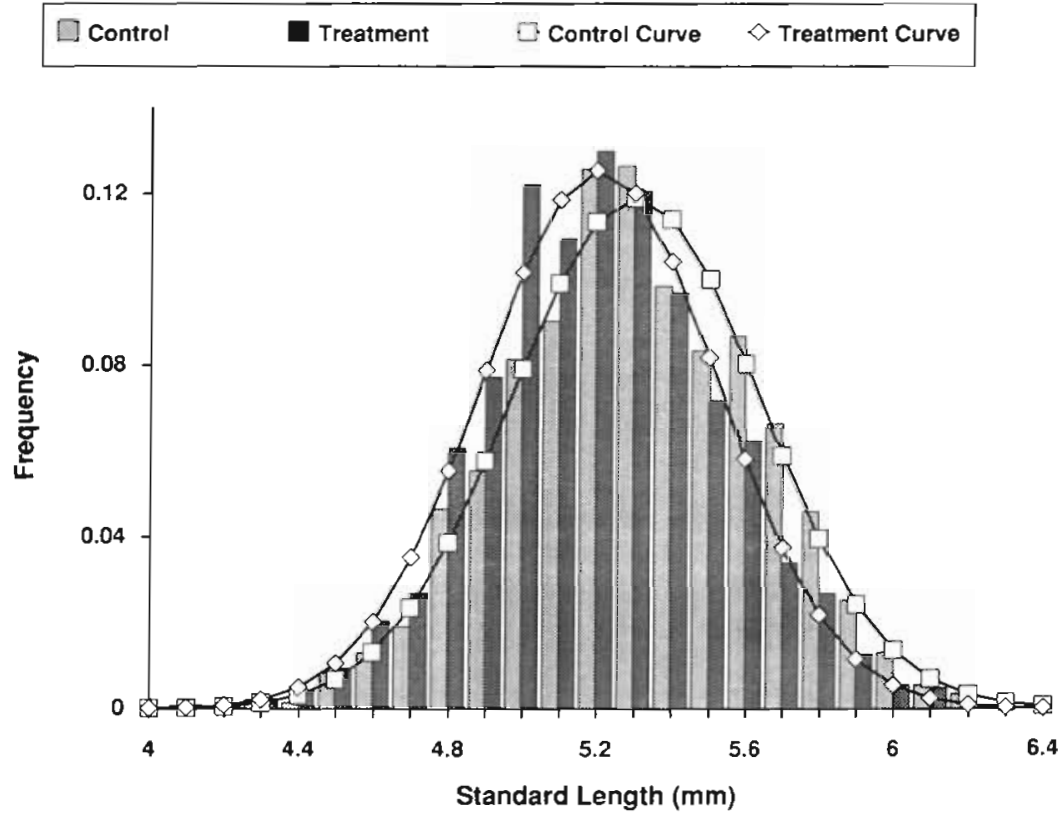

Fig. 3. Gasterosteus aculeatus preying on Mallotus villosus. Pooled size-distributions from mesocosm experiments on the impact of age-selective predation on larval capelin by a stickleback predator. demonstrating the observed reduction in sizes of surviving larvae due to differential predation on older individuals. The overlays are plots of the normal distributions derived from the mean and standard deviation of the control and treatment results used to create the histograms. The fit was tested with a chi-squared goodness of fit test and was found to be highly significant $(p \leqslant 0.001)$ transformation); and (3) the mean size of larvae recovered from each enclosure.

The stickleback predators consumed a significant number of larvae in all treatment enclosures (Table 2). The significant interaction term for the number of larvae recovered reflected a difference in magnitude in the number of larvae eaten in the predator treatments across the experiments, but not a change in direction.

The proportion of larvae classifjed as dead at the termination of the experiments did not differ between controls and treatments ( 33 and $31 \%$ respectively). We concluded that there was no significant larval death as a result of unsuccessful attacks by the stickleback predator

There was a significant difference in the size of the larvae recovered from the treatment and control enclosures. Larvae recovered from the control enclosures were significantly larger than those recovered from the treatment enclosures (Table 2, Fig.
3). This shift in control versus treatment distributions indicates that the sticklebacks consumed more older (larger) than younger larvae.

Coincident with each enclosure trial we reared 100 larvae of the Day 0 and Day 2 cohorts separately in the lab at in situ temperatures. These larvae were preserved alive at the end of the enclosure trials. We found a consistent difference in size between these 2 cohorts at the termination of the experiments. The differences in size of individuals recovered from the control and treatment mesocosm trials can thus be safely interpreted as a consequence of differences in age (as reflected by size).

\section{Jellyfish predators}

All data were normal and homoscedastic. We again tested for a difference between controls and treatments 
for the following variables: (1) the number of larvae recovered; (2) the proportion classified dead at the termination of the experimental trials (after arcsin transformation); and (3) the mean size of larvae recovered from each enclosure.

The jellyfish consumed a significant proportion of the larvae offered (Table 3 ). While the result was not statis-

Table 3. Staurophora mertensi preying on Mallotus villosus. Enclosure age/choice trial with a jellyfish as the predator $t$-test

\begin{tabular}{|lccc|}
\hline Variable & di & Tail & Probability \\
\hline Number recovered & 11 & 1 & 0.018 \\
Mean size & 11 & 1 & 0.006 \\
Percent classified dead (arcsin) & 11 & 1 & 0.096 \\
\hline
\end{tabular}

tically significant there was a suggestion that more larvae were dead at the termination of the treatment versus the control trials, 55 and $46 \%$ respectively. This suggests that some larvae (ca $9 \%$ ) may have died as result of unsuccessful predator attacks.

Jellyfish, too, consumed more large than small larvae (Fig. 4). This shift in the size distribution in predator versus control enclosures reflects the outcome of differential predation against the older larvae

\section{Predation rates}

The mean daily instantaneous mortality rate induced by stickleback predation over the experimental inter- val was $0.52 \pm 0.07$ (SE). If only daylight periods are used in the analysis (reflecting the fact that sticklebacks are visual predators; Ibrahim \& Huntingford 1989), the mean instantaneous rate was $0.89 \pm 0.12$. The mean daily predation rate imposed by sticklebacks on capelin larvae was 246 larvae $d^{-1}$ ( \pm 27, SE) over the full $24 \mathrm{~h}$ period or 352 larvae $\mathrm{d}^{-1}$ ( \pm 35 ) during daylight.

The mean instantaneous daily mortality rate imposed by jellyfish was $0.066 \pm 0.009$ (SE). We consider the increased number of larvae classified as dead at the end of the experiments to be due to unsuccessful predation attempts. Incorporating this difference increased the mean instantaneous daily mortality rate to $0.074 \pm 0.010$. The mean predation rate exerted by

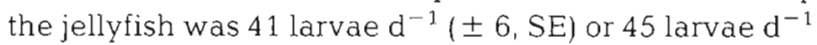
$( \pm 6)$ when the extra larvae believed to have been killed by unsuccessful predation events were included.

\section{Laboratory experiments}

Expt 1: Influence of predator choice on vulnerability of larvae of different ages to predation. The data were normal and homoscedastic. We used a comparison among means ANOVA (Sokal \& Rohif 1981) to compare the mean larval size recovered from treatment versus control tanks for each experimental combination of the age 'cohorts'. The mean size of the larvae recovered from the treatments were smaller (younger) than the those recovered from the controls (Table 4) indicating high predation rates on larger larvae. Fisher's test of combined probabilities indicated this to be a highly significant result. These results were simi-
Fig. 4. Staurophora mertensi preying on Mallotus villosus. Pooled size-distributions from the mesocosm experiment on the impact of age-selective predation on larval capelin by the jellyfish predator. demonstrating the observed reduction in sizes of surviving larvae due to differential predation on older individuals. The overlays are plots of normal distributions derived from the mean and standard deviation of the control and treatment results used to create the histograms. The fit was tested with a chi-squared goodness of fit test and was found to be highly significant $(p \ll 0.001)$

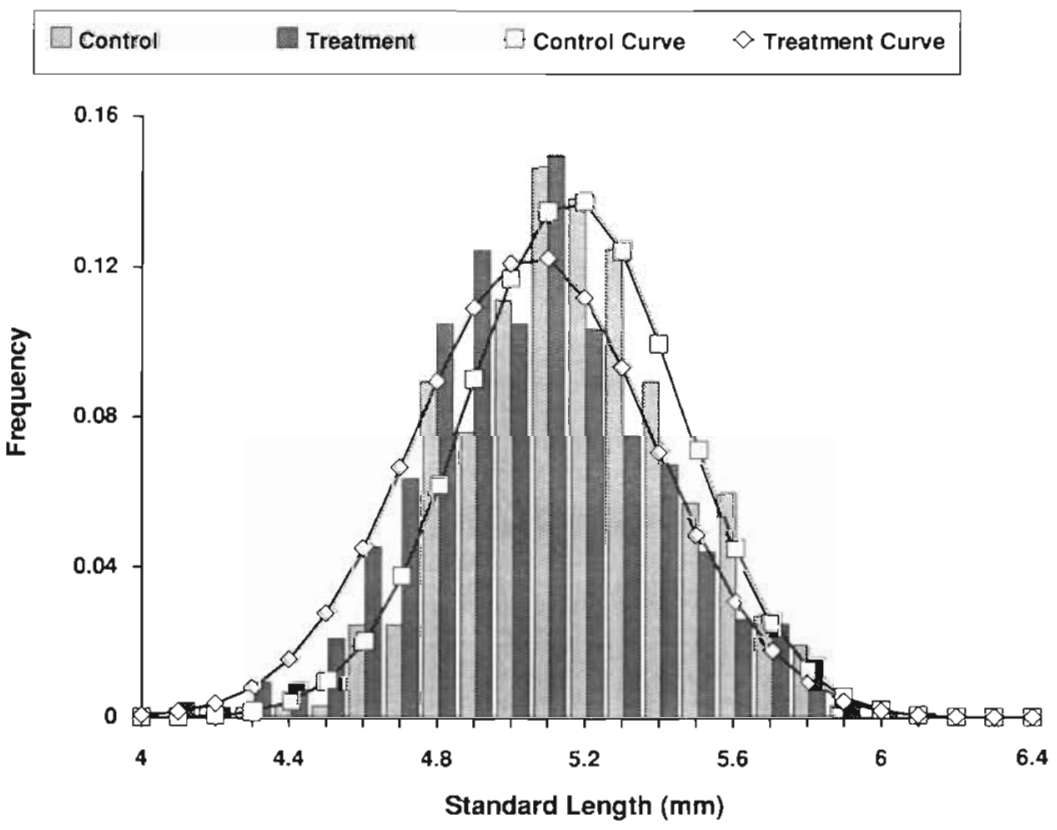


Table 4. Gasterosteus aculeatus preying on Mallotus villosus Lab age/choice trials. Comparison among means ANOVA

\begin{tabular}{|lcc|}
\hline Combination & df & Probability \\
\hline Day 0 \& 2 & 1,261 & 0.08 \\
Day 1 \& 3 & 1,261 & $<0.0001$ \\
Day 2 \& 4 & 1,261 & $<0.0001$ \\
Day 3 \& 5 & 1,261 & 0.013 \\
Day 4 \& 6 & 1,171 & $<0.0001$ \\
Combined probabilities & & $\ll 0.001$ \\
\hline
\end{tabular}

lar in direction and magnitude to those obtained in the enclosure experiments. We can therefore conclude that it is safe to interpret the results from the laboratory experiments without regard for container size effects.

Expt 2: Influence of predator choice on vulnerability of larvae of like age, but different size, to predation. Data were non-normal and heteroscedastic. We used Wilcoxon's signed rank test (Sokal \& Rohlf 1981) to test the influence of size on predator choice. When presented with a choice between different-sized larvae of like age the sticklebacks consumed greater numbers of larger larvae (Table 5). This relationship was consistent over each of the age cohorts tested.

Table 5. Gasterosteus aculeatus preying on Mallotus villosus. Lab size/choice trials. Wilcoxon's signed-rank test

\begin{tabular}{|lc|}
\hline Combination & Probability \\
\hline Day 0 small and large & 0.1 \\
Day 1 small and large & 0.008 \\
Day 2 small and large & 0.004 \\
Day 3 small and large & 0.004 \\
Day 4 small and large & 0.004 \\
Combined probabilities & $\ll 0.001$ \\
\hline
\end{tabular}

Expt 3: Influence of age on the probability of capture. Data were normal and homoscedastic. A 1-way ANOVA was run to test the effect of age on the probability of capture. There was a significant effect of age on the number of larvae consumed per bite $(\mathrm{df}=8, \mathrm{p}=$ 0.007 ). Older larvae had a higher probability of escape following the initiation of a predation event (Fig. 5).

Expt 4: Influence of size, independent of age, on the probability of capture. Data were normal and homoscedastic. We used a 2-way ANOVA on the number of larvae consumed per bite for the 2 size cohorts versus age. There was no interaction effect ( $\mathrm{df}=4, \mathrm{p}=0.61$ ) between age and size. There was also no effect of difference in size ( $\mathrm{df}=1, \mathrm{p}=0.73)$ over age. There was, however, a significant difference in the effect of age on probability of capture $(\mathrm{df}=4, \mathrm{p}=0.02)$. These findings indicate: (1) that there is no difference in the

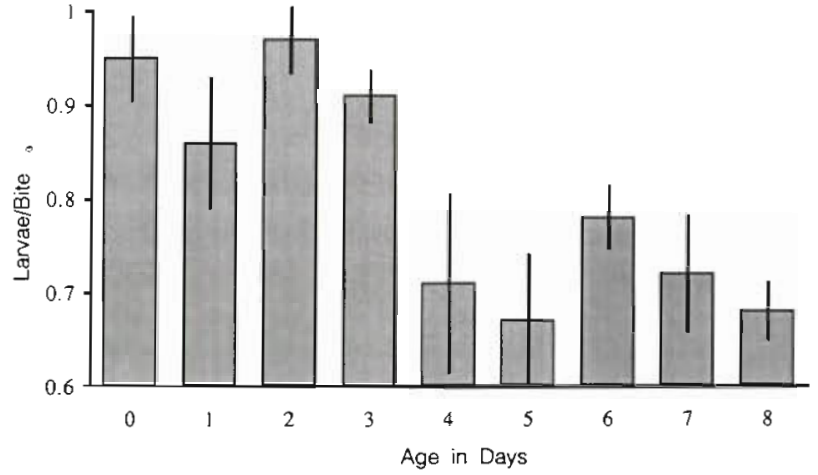

Fig. 5. Gasterosteus aculeatus preying on Mallotus villosus. Probability of capture (larvae eaten per bite) \pm SE for stickleback predators feeding on larval capelin of varying age (Expt 3). Daily cohorts of capelin larvae were subjected to predation trials in the experimental arenas

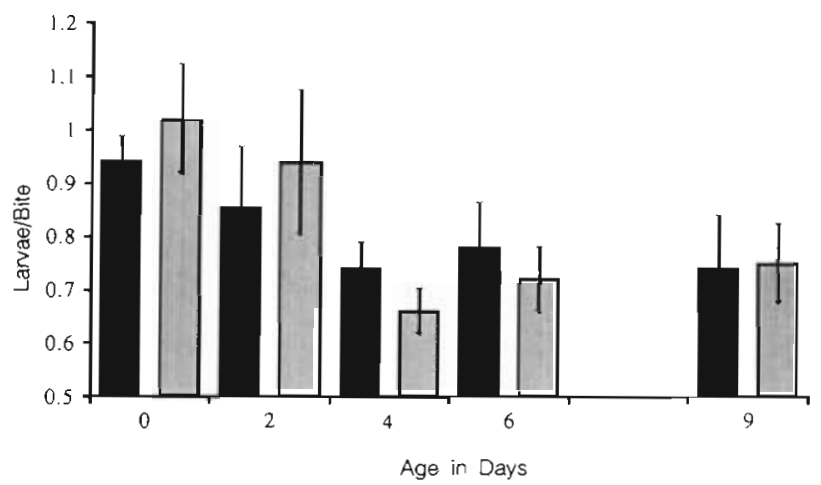

Fig. 6. Gasterosteus aculeatus preying on Mallotus villosus. Probability of capture (larvae eaten per bite) \pm SE for stickleback predators feeding on Iarval capelin of like age but different size [small (black bars), large (shaded bars)] (Expt 4). Daily cohorts of capelin larvae of the 2 different-sized cohorts were separately subjected to predation trials in the experimental arenas

probability of capture of larvae of different size but like age, and (2) that, independent of size, the probability of capture decreases with age (development) (Fig. 6).

\section{DISCUSSION}

\section{Predation rates}

The mortality rates determined from our mesocosm results were very similar to those reported for other in situ enclosure studies of predation on larval fishes (e.g. Gamble \& Houde 1984, Oiestad 1985, de Lafontaine \& Leggett 1987b, Fuiman \& Gamble 1988, Fuiman 1989). This occurred in spite of the fact that different predators, larvae (with the exception of de Lafontaine \& Leggett 1987b), and initial densities of larvae were used. The mean instantaneous mortality rates for fish 
and jellyfish predators of 0.89 (daylight only) and 0.07 represent mortality rates of 55 and $7 \% \mathrm{~d}^{-1}$, respectively. These high predation rates indicate that the high average mortality rate of $60 \% \mathrm{~d}^{-1}$ reported for yolk-sac capelin by Taggart \& Leggett (1987) could be due to predation. The very high mortality rate imposed by the stickleback predators in our mesocosm trials is consistent with Fuiman \& Gamble's (1988) conclusion that there is potential for very high predation rates on fish larvae by piscivorous predators. Thus, in situations where numbers of predators of these types overlap in time and space with fish larvae, the impact on larval mortality could be severe and the potential for phenotypic selection is high.

\section{Age- and size-selective predation}

The results of our probability of capture experiments (no choice: lab Expts 3 and 4) indicate, as expected from the results of earlier studies of this type, that as individual larvae grow/age their probability of escaping a predator when attacked increases. However, probability of capture is only one of the components affecting the vulnerability of larvae to predation. The predation event consists of a series of 3 sequential components: prey encounter, attack and capture. Thus gross vulnerability (GV) to predation (as measured in our mesocosm experiments) can be viewed as the product of the probabilities of: encounter $[P(e)]$; attack [P(a)]; and capture [P(c)] (Fuiman 1989; originally modified from O'Brien 1979).

In our mesocosm studies involving visual predators (sticklebacks), which were presented with a choice of prey of different age, we observed positive ageselective mortality of larvae due to predation. Given that the probability of capture decreased with larval age in our single cohort laboratory experiments, we conclude that the higher mortality experienced by older larvae in the mesocosms resulted from a combined rate of increase in the probabilities of encounter and attack with age that exceeded the observed decline in the probability of capture with age

Two recent studies of predation on marine fish larvae also suggest, as we found, that older larvae may, under certain conditions, be more vulnerable to predation. Fuiman (1989) reported that, in enclosure experiments on the vulnerability of herring larvae Clupea harrengus to predation, older larval cohorts (no-choice experiments) experienced a higher mortality rate when in the presence of a fish predator. This finding was contrary to his laboratory findings where younger cohorts experienced higher mortality. He hypothesized that the greater vulnerability of older larvae to predation reflected the importance of differential age-related changes in the probabilities of encounter and attack.

Hoenig et al. (1990) reanalyzed the data of Creco et al. (1983) on mortality rates of larval American shad Alosa sapidissima in the Connecticut River and concluded that younger (late-hatching) larvae enjoyed higher survival rates than did older larvae (earlyhatched). These results, too, suggest that vulnerability of shad larvae to the agents of mortality (transport, predation, etc.) increased with age. However, they did not speculate on the agent of mortality.

Several factors could contribute to the greater overall vulnerability of older larvae to visual predators, as observed in our studies, and by Fuiman (1989) and Hoenig et al. (1990). Encounter rates may increase with age as a consequence of the greater swimming speeds and encounter radii of older larvae. In our particular study, however, the swimming speed of the larval prey relative to that of the stickleback was very small. Hence, in this prey/predator pairing we hypothesize that the change in the probability of encounter due to increased swimming speeds is minimal. Encounter rates could, however, increase with larval age as a consequence of increases in the reactive field of the predator for larvae of greater age and size (Gerritsen \& Strickler 1977, O'Brien 1979). O'Brien (1979) reported that an increase in the size of Daphnia sp. from 1 to $2 \mathrm{~mm}$ caused a 27 times increase in the probability of detection.

For visual predators, increased coloration and movement (which in the case of larval fish are more commonly associated with age/development) can also play a role in increasing the reactive distance of predators to prey and hence increasing encounter rate (Bailey \& Houde 1989). In this connection Ibrahim \& Huntingford (1989) examined the role of visual cues on prey selection in the 3-spined sticklebacks. Several of their findings are relevant to the results we observed. First, larger prey items were preferred over smaller prey and, second, prey which exhibited greater movement were attacked more frequently.

The probability of attack can also increase as a result of differences in prey behavior and morphology. Foraging theory suggests that a predator should selectively consume prey which minimize the energy cost/benefit ratio (see Stephans \& Krebs 1986 for a review). Therefore older larvae may be preferred, even though their probability of escape is greater than that of younger individuals.

In our enclosure trials involving jellyfish we again observed higher predation rates on older versus younger larvae. For raptorial ambush predators such as jellyfish the probabilities of attack should approximate unity since such predators are likely to attack any larvae they contact. It is likely, too, that the probability 
of capture by jellyfish, once encountered, will decrease with age (as was the case in our laboratory experiments with sticklebacks) as larvae become better able to avoid contacts and escape attacks (Baily \& Batty 1983. 1984). We conclude, therefore, that the increase in predation rates on older larvae, as observed in the mesocosm experiments involving jellyfish predators, could have occurred because the rate of increase in larval encounters with jellyfish with age [resulting largely from increases in prey target size (greater contact surface area) and swimming speeds of the larvae themselves] exceeded the rate of decline in the probability of capture with age.

Bailey \& Houde (1989), following Greene's (1986) approach, have recently proposed a suite of conceptual models which combine predator encounter rate and prey susceptibility throughout development. In these models, susceptibility to predation is seen as product of probabilities of attack and capture (the proportion of encounters which result in the ingestion of the prey) (Greene 1986). These models suggest that larvae preyed upon by ambush raptorial invertebrate and raptorial fish predators should exhibit vulnerability curves which are dome-shaped when plotted with respect to age. While we did not observe this domeshaped relationship in our results, the range of larval ages we examined was restricted to the first few days of life (when, it is important to note, larval mortality rates appear to be the highest). It is quite possible that, as larvae age and grow beyond the stages we investigated, they will experience a decrease in vulnerability to these predators as predicted by the models. In partial support of the hypothesized dome-shaped response, Pepin et al. (1992, this issue) observed a reduction in the impact of fish predators with increased mean larval size between mesocosm trials. However, within trials, larger larvae suffered greater mortality due to predation as was the case in our experiments (Pepin et al. 1992, this issue).

With the exception of the experiments we report here, there have been no studies on the impact of variability in size within an age cohort on the vulnerability of larval fish to predation. Our laboratory experiments on size effects, independent of age effects, indicate that smaller larvae had the same probability of escape $[1-P(c)]$ as large individuals of the same cohort. Yet, when the visual predators used in our experiments were presented with a choice between large and small larvae of the same age they consistently selected larger larvae. We have already noted (in our treatment of age effects) that a small difference in size can have a dramatic effect on the probability of encounter and attack. These size-based arguments also hold for the influence of size within an age cohort on the vulnerability of larvae to predation. Thus we expect larger

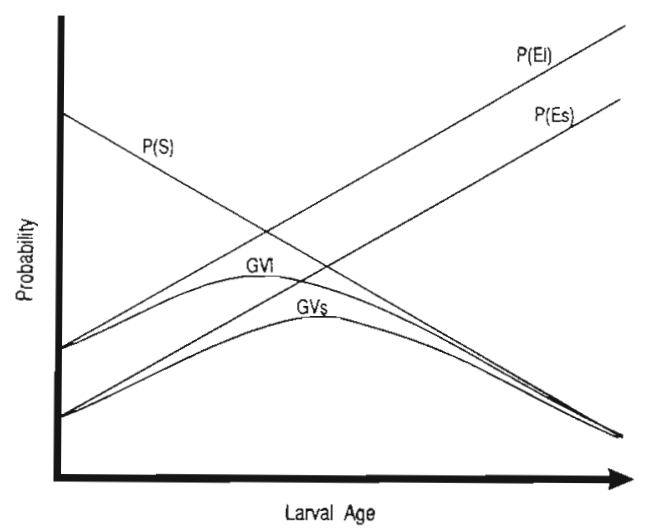

Fig. 7. Conceptual model of the influence of age-related changes in encounter rates on the gross vulnerability of larval fish of different sizes to predation, where $\mathrm{P}(\mathrm{Es})=$ probability of encounter for small larvae; $\mathrm{P}(\mathrm{El})=$ probability of encounter for large larvae $; \mathrm{P}(\mathrm{S})=$ susceptibility of both large and small larvae to predation (combined probability of attack and capture): $G V s=$ gross vulnerability of small larvae to predation; and GVI = gross vulnerability of large larvae to predation

larvae to be encountered at a higher frequency and, assuming they represent a more desirable prey for the predator, experience a higher probability of attack.

The combined evidence presented above suggests that, contrary to the 'bigger-is-better' hypothesis as it relates to the survival probabilities of larval fishes, being smaller at a given age could, under some conditions, and at certain stages of larval development, confer a survival advantage. The decreased probabilities of encounter and attack characteristic of smaller larvae may, in fact, provide a refuge from predation at some stages of development. This result is illustrated in Fig. 7 in which the probabilities of encounter, susceptibility (product of probabilities of attack and capture) and vulnerability for large and small larvae are plotted as a function of age. The higher overall vulnerability of intermediate-sized larvae and the resultant gross vulnerability to predation revealed in this hypothesized example, and in our data, are far from unique in aquatic communities. Brooks \& Dodson (1965) provided the first of many examples of size-selective predation against bigger species and bigger individuals within species of zooplankton (for a review see Stein et al. 1988).

The results of our experiments, their consistency with the results of recent studies on larval fishes (Fuiman 1989. Hoenig et al. 1990, Pepin et al. 1992), and the more extensive body of literature on size-selective predation on freshwater zooplankton strongly suggest that a detailed review of 'the bigger-is-better' hypothesis, as it relates to anticipated survival rates in larval fishes, is in order Based on our findings to date, we hypothesize that the basis for important deviations 
from this expectation will be found in a quantitative understanding of the dynamic interaction between size- and age-specific rates of encounter, attack, and capture, which together determine size- and agespecific vulnerabilities to predation.

Acknowledgements: We are indebted to the following people for help during the field and lab phase of this work: Year $1-$ C. Chambers, E. Fells, A. Ferron, G. Henry, G. Maillett, M. C. Morrison, and K. Tallon; Year 2 - K. Barry, E. Demers, C. duSouiche, D. Hamilton, H. Kirschner, M. Krohn, T Herra, and P. Simon. We also thank W.C.L's lab members for their help in the developmental phase of this research: A. Appenzellar, D. Bertram, C. Chambers, E. Deblois, A. Ferron, B. Mackenzie, and T. Miller Thanks also go to $\mathrm{H}$. Browman for introducing us to the silhouette videographic technique and to J. Brown, J. Carscadden and B. Nakashima for their logistical help in Newfoundland. Finally we would like to thank the residents of Bryant's Cove, Newfoundland for their interest in our work and their help in ensuring that it succeeded. This research was supported by an NSERC operating grant and a DFO subvention to W.C.L.

\section{LITERATURE CITED}

Baily, K. M., Batty, R. S. (1983). A laboratory study of predation by Aurelia aurita on larval herring (Clupea harengus). Mar Biol. 72: 295-301

Baily, K. M., Batty, R. S. (1984). Laboratory study of predation by Aurelia aurita on larvae of cod, flounder, plaice and herring: development and vulnerability to capture. Mar. Biol. 83: 287-291

Bailey, K. M. Houde, E. D. (1989). Predation on eggs and larvae of marine fishes and the problem of recruitment. Adv mar. Biol 25: 1-83

Blaxter, J. H. S. (1986). Development of sense organs and behaviour of teleost larvae with special reference to feeding and predator avoidance. Trans. Am. Fish. Soc. 115: $98-114$

Blaxter, J. H. S., Batty, R. S. (1985). The development of startle responses in herring larvae. Mar. biol. Ass. U.K. 65: $737-750$

Butler, J. L., Pickett, D. (1988). Age-specific vulnerability of Pacific sardine, Sardinops sagax, larvae to predation by northern anchovy. Fish. Bull. U.S. 86: 163-167

Brooks, J. L., Dodson, S. I. (1965). Predation, body size and composition of plankton. Science 150: 503-512

Chambers, C. C., Leggett, W. C., Brown, J. A. (1989). Egg size, maternal effects, and the correlations between early life history traits of capelin Mallotus villosus (Pisces: Osmeridae): an appraisal at the individual level. Fish. Bull. U.S. $87 \cdot 515-523$

Creco, V T., Savoy, T., Gunn, L. (1983). Daily mortality rates of larval and juvenile American shad (Alosa sapidissima) in the Connecticut River with changes in year-class strength. Can. J. Fish. Aquat. Sci. 40: 1719-1728

de Lafontaine, Y., Leggett, W. C. (1987a). Evaluation of in situ enclosure studies for larval fish studies. Can. J. Fish. Aquat. Sci. 44: 54-65

de Lafontaine, Y., Leggett, W. C. (1987b). Effect of container size on estimates of mortality and predation rates in experiments with macrozooplankton and larval fish. Can. J. Fish. Aquat. Sci. 445: 1173-1190

de Lafontaine, Y., Leggett, W. C. (1988). Predation by jellyfish on larval fish: an experimental evaluation employing in situ enclosures. Can J. Fish. Aquat. Sci. 45: 1173-1190

Eaton, R. C., DiDomenico, R. (1986). Role of the Teleost escape response during development. Trans. Am. Fish. Soc. 115: $128-142$

Fancett, M. S., Jenkins, G. P. (1988). Predatory impact of scyphomedusae on icthyoplankton and other zooplankton in Port Phillip Bay. J. exp. mar Biol. Ecol. 116: 63-77

Folkvord, A., Hunter, J. A. (1986). Size-specific vulnerability of northern anchovy, Engraulis mordax, larvae to predation by fishes. Fish. Bull. U.S. $84: 859-870$

Frank, K. T, Leggett, W C. (1981a). Wind regulation of emergence times and early larval survival in capelin (Mallotus villosus). Can. J. Fish. Aquat. Sci. 38: 215-223

Frank, K. T., Leggett, W C. (1981b). Prediction of egg development and mortality rates in capelin (Mallotus villosus) from meteorological, hydrographic, and biological factors. Can. J. Fish. Aquat. Sci. 38: 1327-1338

Frank, K. T., Leggett, W. C. (1982). Environmental regulation of growth rate, efficiency, and swimming performance in larval capelin (Mallotus villosus), and its application to the match/mismatch hypothesis. Can. J. Fish. Aquat. Sci. 39 $691-699$

Fuiman, L. A. (1989). Vulnerability of Atlantic herring larvae to predation by yearling herring. Mar Ecol. Prog. Ser. 51 291-299

Fuiman, L. A., Gamble, J. C. (1988). Predation by Atlantic herring, sprat, and sandeels on herring larvae in large enclosures. Mar Ecol. Prog. Ser. 44:1-6

Gamble, J. C., Houde, E. D. (1984). Growth, mortality and feeding of cod (Gadus morhua L. I larvae in enclosed water columns and in laboratory tanks. In: Dahl, E. Danielssen, D. S., Moksness, E., Solemdal, P. (eds.) The propagation of cod Gadus morhua L. Part 1. Institute of Marine Research, Flødvegan Biological Station, Arendal, Norway, p. 123-143

Gerritsen, J., Strickler, J. R. (1977). Encounter probabilities and community structure in zooplankton: a mathematical model. J. Fish. Res. Bd Can. 34: 73-82

Greene, C. H. (1986). Patterns of prey selection: Implications of predator foraging tactics. Am. Nat. 128: 824-839

Hewitt, R. P., Theilacker, G. H., Lo, N. C. H. (1985). Causes of mortality in young jack mackerel. Mar. Ecol. Prog. Ser. 28: $1-10$

Hoenig, J. M., Pepin, P., Lawing, W. D. (1990). Estimating relative survival rate for two groups of larval fishes from field data: do older larvae survive better than young? Fish. Bull. U.S. 88: 485-491

Hunter, J. R. (1984). Inferences regarding predation on the early life stages of cod and other fishes. In: Dahl, E., Danielssen, D. S., Moksness, E., Solemdal, P. (eds.) The propagation of cod Gadus morhua L. Part 1. Institute of Marine Research, Flodvegan Biological Station, Arendal, Norway, p. 533-562

Ibrahim, A. A., Huntingford, F. A. (1989). The role of visual cues in prey selection in three-spined sticklebacks (Gasterosteus aculeatus). Ethology 81: 265-272

Leggett, W. C., Frank, K. T., Carscadden, J. E. (1984). Meteorological and hydrographic regulation of year-class strength in capelin (Mallotus villosus). Can. J. Fish. Aquat. Sci. 41: 1193-1201

Margulies, D. (1989). Size-specific vulnerability to predation and sensory development of white seabass, Atracosin nobilis, larvae. Fish. Bull. U.S. 87: 537-552

McGurk, M. D. (1986). Natural mortality of marine pelagic fish eggs and larvae: role of spatial patchiness. Mar. Ecol. Prog Ser. 34: 227-242

Miller, T. J., Crowder, L. B., Rice, J. A., Marschall, E. A. (1988) 
Larval size and recruitment mechanisms in fishes: toward a conceptual framework. Can. J. Fish. Aquat. Sci. 45: $1657-70$

O'Brien, W. J. (1979). The predator-prey interaction of planktivorous fish and zooplankton. Am. Scient. 67: 572-581

Oiestad, V. (1985). Predation on fish larvae as a regulatory force, illustrated in mesocosm studies with large groups of larvae. N. Atlant. Fish. Orgn (NAFO) sci. Coun. Stud. 8: 25-32

Pepin, P., Shaers, T H., de Lafontaine, Y (1992). The significance of body size to the interaction between a larval fish (Mallotus villosus) and a vertebrate predator (Gasterosteus aculeatus). Mar. Ecol. Prog. Ser. 81: 1-12

Radtke, R. L. (1990). Larval fish age, growth, body shrinkage: information available from otoliths. Can. J. Fish. Aquat. Sci. 46: $1884-1894$

Rogers, D. J. (1972). Random search and insect population models. J. Anim. Ecol. 41: 369-383

Royama, T (1971). A comparative study of models of predation and parasitism. Res. Popul. Ecol. Suppl. 1 1-91

This article was submitted to the editor
Sokal, R. R., Rohlf, F. J (1981). Biometry, 2nd edn. W. H. Freeman \& Co., San Francisco

Stein, R. A., Threlkeld, S. T., Sandgren, C. D., Sprules, W. G., Persson, L., Werner, E. E., Neill, W. E., Dodson, S. I. (1988). Size structured interaction in lake communities. In: Carpenter, S. R. (ed.) Complex interactions in lake communities. Springer-Verlag, New York, p. 161-179

Stephens, D. W., Krebs, J. R. (1986). Foraging theory. Princeton University Press, Princeton

Taggart, C. T., Leggett, W C. (1987). Short-term mortality in post-emergent larval capelin Mallotus villosus. I. Analysis of multiple in situ estimates. Mar. Ecol. Prog. Ser. 41: 205-217

Ware, D. M. Lambert, T. C. (1985). Early life history of Atlantic mackerel (Scomber scombrus) in the southern Gult of St. Lawrence. Can. J. Fish. Aquat. Sci. 42: 577-592

Webb, P. W., Weihs, D. (1986). Functional locomotor morphology of early life history stages of fishes. Trans. Am. Fish. Soc. 115: 115-127

Manuscript received: June 5, 1991

Revised version accepted: February 14, 1992 